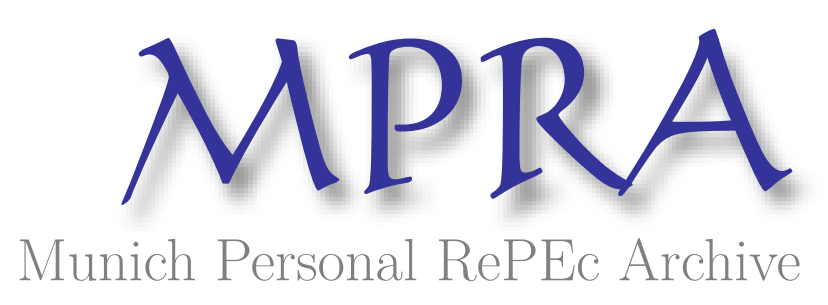

\title{
Bubbles, shocks and elementary technical trading strategies
}

\author{
Fry, John
}

University of Sheffield

15 May 2013

Online at https://mpra.ub.uni-muenchen.de/47052/

MPRA Paper No. 47052, posted 17 May 2013 14:57 UTC 


\title{
Bubbles, shocks and elementary technical trading strategies
}

\author{
John Fry*
}

May 2013

\begin{abstract}
In this paper we provide a unifying framework for a set of seemingly disparate models for bubbles, shocks and elementary technical trading strategies in financial markets. Markets operate by balancing intrinsic levels of risk and return. This seemingly simple observation is commonly over-looked by academics and practitioners alike. Our model shares its origins in statistical physics with others. However, under our approach, changes in market regime can be explicitly shown to represent a phase transition from random to deterministic behaviour in prices. This structure leads to an improved physical and econometric model. We develop models for bubbles, shocks and elementary technical analysis strategies. We apply our model to real-estate bubbles and to the on-going Eurozone crisis. We close by comparing the results of our mathematical model with the results of qualitative analyses from the finance literature.
\end{abstract}

\section{Introduction}

The analogy between financial crashes and phase transitions in critical phenomena in statistical physics is now well established (Johansen et al., 2000; Sornette 2003). A vast literature discusses the hitherto somewhat controversial subject of log-periodic precursors to financial crashes (see e.g. Zhou and Sornette (2008, 2009); Feigenbaum (2001a-b); Chang and Feigenabum (2006, 2008); Bree and Joseph, 2013). UK.

${ }^{*}$ The University of Sheffield, Management School, Conduit Road, Sheffield S10 1 FL,

E-mail: John.Fry@sheffield.ac.uk 
Financial markets operate by balancing risk and return (Markowitz, 1971). As discussed in Fry (2012) there is a sense in which the prevailing class of log-periodic models omits a crucial second-order condition pertaining to the inherent riskiness of financial markets. There is thus an interesting sense in which the academic literature mirrors the popular failings of market players prior to the 2008 crisis (Peston and Knight, 2012) in terms of an inability to identify both market over-confidence and the intrinsic riskiness involved. Despite its genesis in statistical physics - log-periodic and related models have begun to permeate into the mainstream financial literature and have thus clearly achieved an element of wider significance (see e.g. Lin and Sornette, 2011; Geraskin and Fantazzini, 2011; Bree et al., 2013; Kurz-Kim, 2012; Yan et al., 2012; Jiang et al., 2010). However, despite obvious merit these works have the inescapable drawback of representing increasingly sophisticated attempts to fit a fundamentally flawed model. Here, in contrast, we can show that a better understanding of the underlying market mechanisms, and a better physical model, leads to significant improvements.

One of the key themes explored by log-periodic models is the prediction of market bubbles and crashes. However, this predictive aspect has been significantly expanded upon in recent years (see e.g. Yan et al., 2012; Sornette and Zhou, 2006). Themes discussed include antibubbles (Zhou and Sornette; 2004, 2005) and the effects of external shocks like the 9/11 terror attacks upon financial markets (Sornette et al., 2003; Lillo and Mantegna, 2003; Johansen and Sornette, 2010; Fry, 2012). Work in Fry (2012) united a set of previously disparate models for endogenous and exogenous market crashes. Here, we show that this approach can be extended and that this has dramatic consequences. In particular, we find ourselves in the exciting position of being able to use tools and techniques from statistical physics to forge links between academic and practitioner finance (Pan et al., 2006).

The academic literature on technical analysis is voluminous (see e.g. Park and Irwin, 2007). Amidst a broad range of findings a majority of papers report that some technical trading rules appear to be profitable. Even if the profitability of technical analysis is open to debate, such methods may provide genuinely useful information (Lo et al., 2000; Osler, 2000). As in Pan et al. (2006) there are clear links with our model and technical trading strategies (see e.g. Murphy, 1986). There are obvious parallels between endogenous and exogenous market shocks and the information shocks identified in Plummer (2006). Moreover, we develop methods to identify cycles and support and resistance lines commonly studied by practitioners (see e.g. Plummer, 2006; Griffis and Epstein, 2009).

Beyond narrow academic interests our contribution is both timely and 
relevant. Firstly, we are able to use our model to detect bubbles and antibubbles in UK house prices. Real estate bubbles in the USA and throughout Europe have played a key role in recent crises. Secondly, we are able to show that antibubbles and endogenous shocks lie at the heart of the Greek crisis in the Eurozone (Pryce, 2012). Thirdly, we are able to derive quantitative results to cross-validate purely qualitative findings in the financial literature.

The layout of this paper is as follows. Section 2 presents our basic modelling approach. Markets are assumed to work by balancing intrinsic levels of risk and return. Once this feature is understood bubbles and crashes, and other phenomena, can be modelled as temporary departures from longterm equilibria. In Section 3 we model bubbles and antibubbles. In Section 4 we discuss information shocks and in relation to the on-going European sovereign debt crisis. Sections 5-6 cover further extensions of market predictions relative to the practitioner literature. In Section 5 we model cycles. In Section 6 we model support and resistance lines. Finally, Section 7 concludes.

\section{The model}

Markets are assumed to work by balancing the level of risk and the rate of return. The level of risk and return remain fixed even in the face of technological innovation or an influx of new investors (Zeira, 1999). These assumptions do not rely on complicated mathematics and so avoid dubious assumptions such as the "riskless hedge" of the Black-Scholes model (Bouchaud and Potters, 2003). Our model makes several observable predictions for market crashes. Endogenous and exogenous crashes, cycles and price-level shocks all exhibit identifiable short-run deviations from long-term equilibria. Let $P_{t}$ denote the price of an asset at time $t$ and let $X_{t}=\log P_{t}$. The set up of the model is as follows

Assumption 1 (Intrinsic Rate of Return) The intrinsic rate of return is assumed constant and equal to $\mu$ :

$$
E\left[X_{t+\Delta}-X_{t} \mid X_{t}\right]=\mu \Delta+o(\Delta) .
$$

Assumption 2 (Intrinsic Level of Risk) The intrinsic level of risk is assumed constant and equal to $\sigma^{2}$ :

$$
\operatorname{Var}\left[X_{t+\Delta}-X_{t} \mid X_{t}\right]=\sigma^{2} \Delta+o(\Delta) .
$$




\section{Bubbles and antibubbles}

In this section we show how the framework laid out by the equations (1-2) can be used to generate a model for financial bubbles discussed in $\operatorname{Fry}(2010 \mathrm{a}$ b), (2011), (2012) extending a deterministic version of the same model in Johansen et al. (2000) and a series of later papers, see Section 1, which all omit a critical second-order condition given by equation (11). Let $P(t)$ denote the price of an asset at time $t$. Our starting point is the equation

$$
P(t)=P_{1}(t)(1-\kappa)^{j_{1}(t)},
$$

where $P_{1}(t)$ satisfies

$$
d P_{1}(t)=\left(\mu(t)+\sigma^{2}(t) / 2\right) P_{1}(t) d t+\sigma(t) P_{1}(t) d W_{t},
$$

where $W_{t}$ is a Wiener process and $j_{1}(t)$ is a Poisson process. Taking logs gives

$$
d X_{t}=\mu(t) d t+\sigma(t) d W_{t}-v d j_{1}(t),
$$

where $v=-\ln [1-\kappa]>0$.

Fundamental price/equilibrium/benchmark IID model. Suppose that the intensity of the Poisson process $j_{1}(t)$ is constant:

$$
\lambda(t)=\lambda .
$$

Equation (6) shows that incoporating background price risks - associated with the probability of a crash - gives a financial model with skewness and heavy tails (see below). Further equation (6) also leads to the intuitively appealing representation of prices as compound interest plus random noise - further punctuated by a random sequence of larger demand shocks. Assumptions 1 and 2 give

$$
\mu(t)=\mu+v \lambda ; \sigma^{2}(t)=\sigma^{2}-v^{2} \lambda .
$$

The model in (6) thus leads to a special case of Merton's classical model (Merton, 1976). The returns distribution corresponding to (5-6) is a GaussianPoisson convolution with probability density

$$
f_{X}(x)=\sum_{n=0}^{\infty} \frac{e^{-\lambda} \lambda^{n}}{n !} \frac{1}{\sqrt{2 \pi} \sqrt{\sigma^{2}-v^{2} \lambda}} e^{-\frac{(x+v n-\mu-v \lambda)^{2}}{2\left(\sigma^{2}-v^{2} \lambda\right)}} .
$$

The model in (8) has wider significance as a financial model (see below). Further, the random variable $X$ in (8) matches the negative skew and excess 
kurtosis of empirical asset returns distributions (Cont and Tankov, 2004). We note that

$$
\begin{aligned}
\operatorname{Skew}(X) & =\frac{E\left[(X-\mu)^{3}\right]}{\sigma^{3}}=-\lambda v^{3}<0, \\
\operatorname{Kurtosis}(X) & =\frac{E\left[(X-\mu)^{4}\right]}{\sigma^{4}}=3+\lambda v^{4}>3 .
\end{aligned}
$$

As an illustration we fit the model in (8) to a time series of log-returns from the S\& P 500 series from 3/1/1950-29/1/2013. The results are shown below in Table 1. The Gaussian-Poisson model offers a significant improvement over the Gaussian model - especially when the returns series is calculated over a shorter time horizon.

\begin{tabular}{|l|l|l|l|}
\hline Interval & Daily & Weekly & Monthly \\
\hline Gaussian & 50896 & 8054.352 & 1320.699 \\
\hline Gaussian-Poisson convolution & 51789.44 & 8176.599 & 1343.364 \\
\hline
\end{tabular}

Table 1: Results: maximised log-likelihood for empirical financial data.

Speculative bubble. We can extend the Gaussian model in Fry (2012) using

$$
\lambda(t)=\lambda+\frac{\beta t^{\beta-1}}{\alpha^{\beta}+t^{\beta}} .
$$

In equation (6) the probability of a crash is assumed to remain constant. Here, in contrast, in equation (10) it is time variation in the probability of a crash that characterises the speculative bubble (Johansen et al., 2000). Thus, we are led to statistical tests for bubbles (Section 3.2) and a more elegantly formulated non-Gaussian model than that in Fry (2011). This is significant for empirical applications as accounting for non-Gaussian behaviour leads to more refined tests for speculative bubbles (Fry, 2008).

In the sequel combining equations (7) and (10) gives

$$
\mu(t)=\mu+v \lambda(t) ; \sigma^{2}(t)=\sigma^{2}-v^{2} \lambda(t) .
$$

Equation (11) shows that as in Fry (2012) prior to the (first) crash prices undergo a rapid price rise described by $\mu(t)$ coupled with a temporary and illusory decrease in volatility described by $\sigma^{2}(t)$ - the hallmark of market over-confidence. As $\sigma^{2}(t)$ in (11) goes to zero we can see that in this model 
crashes occur as a result of a phase transition from random to deterministic behaviour in prices.

Anti-bubble. As in Yan et al. (2012) we can model anti-bubbles as the mirror image of conventional asset bubbles by taking $v=-v$ in equations (5-11). Thus, during an anti-bubble regime

$$
\mu(t)=\mu-v \lambda(t) ; \sigma^{2}(t)=\sigma^{2}-v^{2} \lambda(t) .
$$

Similarly, equation (12) shows that antibubbles also occur as a result of a phase transition from random to deterministic behaviour in prices.

\subsection{Empirical Analysis}

In this section we apply the model for bubbles and antibubbles to empirical data on real UK house prices from 1983 to 2011. From equations (7) and (10) we see that testing the null hypothesis of no speculative bubble (resp. no antibubble) is a test of the hypothesis that $\beta=0$. This can be tested using a maximum likelihood ratio test.

A plot of real UK house prices is shown in Figure 1 and suggests that UK house prices are intrinsically volatile and have been subject to various bubble and antibubble episodes over the years. Using Figure 1 we divide the data into sub-samples shown in Table 1 and test for bubbles and antibubbles. Evidence of bubbles in UK house prices is found over the years 1983-1989 and 1999-2006. Evidence for antibubbles is found over the years 1990-96 and 2008-11. Results thus appear to be in reasonable agreement both with Figure 1 and with comparable findings using a Gaussian model in White and Fry (2013).

\subsection{Further extensions: motivation}

Yan et al. (2012) uses the concepts of bubbles and anti-bubbles to predict market rebounds. Here, we show that our model can do this and more besides. Inter alia our extra level of generality allows us to predict cycles and resistance/support lines - leading to a potential union of the academic and practitioner literature on finance. In the next section we discuss information shocks in the context of the on-going European sovereign debt crisis.

\section{Information shocks and the sovereign debt crisis}

The effect of information shocks upon markets is discussed both by the practitioner (Plummer, 2006; Murphy, 1986) and the academic literature (Sor- 


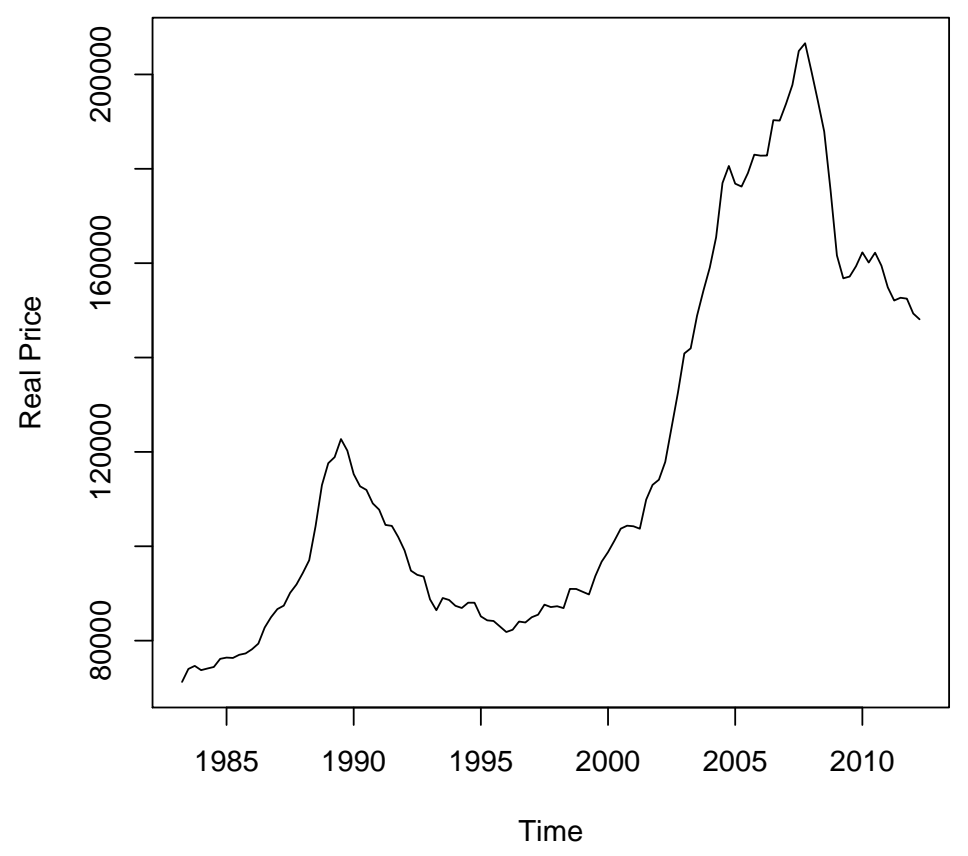

Figure 1: UK average real house prices 1983-2011.

nette et al., 2003; Johansen and Sornette, 2010; Fry, 2012). These latter papers consider the impact of dramatic world-news events like the 9/11 terror attacks, the attempted coup against Soviet President Mikhail Gorbachev in 1991 and the Lehman Brother's bankruptcy upon markets. Volatility is shown to decay following the exogenous shock at a rate quicker that is both faster than after a corresponding endogenous shock (bubble-induced crash) and in such a way that can be distinguished from the background noise. However, information shocks are not limited to such (extreme) external events and Plummer (2006), Chapter 22 describes information shocks as price movements that occur in the context of bigger trends. This includes, but is not limited to, the bubbles and antibubbles discussed above.

In the sequel we develop additional models for endogenous and exogenous information shocks. The discussion is then explicitly tied to the on-going Eurozone crisis with an application to Greek government bond yields. 


\begin{tabular}{|l|l|l|l|l|l|}
\hline Period & $\begin{array}{l}\text { Baseline } \\
\text { Model }\end{array}$ & $\begin{array}{l}\text { Bubble/ } \\
\text { Anti-bubble model }\end{array}$ & $\chi^{2}$ & $p$-value & Conclusion \\
\hline $1983-89$ & 69.28098 & 74.80442 & 11.047 & 0.001 & Bubble \\
$1990-96$ & 60.58248 & 63.29616 & 5.427 & 0.020 & Anti-bubble \\
$1997-02$ & 59.18538 & 62.38472 & 6.399 & 0.011 & Bubble \\
$1998-03$ & 63.12964 & 63.56443 & 0.870 & 0.351 & No bubble \\
$1999-04$ & 59.70104 & 63.43649 & 7.471 & 0.006 & Bubble \\
$2000-05$ & 57.80827 & 62.49827 & 9.38 & 0.02 & Bubble \\
$2001-06$ & 58.09862 & 62.06083 & 7.924 & 0.005 & Bubble \\
$2002-07$ & 57.66191 & 59.27685 & 3.230 & 0.072 & No Bubble \\
$2008-11$ & 34.96031 & 37.19223 & 4.464 & 0.035 & Anti-bubble \\
\hline
\end{tabular}

Table 2: Results of the test for bubbles and anti-bubbles in UK house prices.

\subsection{A model for exogenous market shocks}

Suppose that the market is exposed to an exogenous shock. The shock is assumed to be completely unpredictable but its affect is merely transitory (Crane and Sornette, 2008; Saichev and Sornette, 2010; Sornette and Helmsetter, 2003). The shock occurs at time 0 and results in an initial decrease in drift by the amount $\mu_{0}$ and an initial increase in volatility by the amount $\sigma_{0}^{2}$. As an arbitrage opportunity has to be eliminated, the market recovers at the random time $t_{0}$ - the drift increases by $\mu_{0}$ and volatility decreases by $\sigma_{0}^{2}$. The time $t_{0}$ of the market recovery is a random variable with hazard function $h(t)$. Since the effect of the exogenous shock is transitory it follows that $h^{\prime}(t)>0$, since as time progresses a market rebound becomes increasingly likely. Also, since the shock is assumed to happen at $t=0$ it follows that we must also have $h(0)=0$ :

$$
h^{\prime}(t)>0 ; \quad h(0)=0 .
$$

The price dynamics prior to the market recovery are described by

$$
d X_{t}=\mu(t) d t+\sigma(t) d W_{t}-v d j_{1}(t)+d j_{2}(t)
$$

where, as before, $j_{1}(t)$ is a Poisson process representing ever-present background risks and $j_{2}(t)$ satisfies

$$
d j_{2}(t)=\mu_{0} \delta\left(t-t_{0}\right) d t+i \sigma_{0} \delta\left(t-t_{0}\right) d W_{t}
$$


where $i=\sqrt{-1}$ and $\delta(\cdot)$ denotes Dirac's delta function. When a recovery happens, the effect is an increase in drift and a decrease in the variance, hence the introduction of $i=\sqrt{-1}$. Prior to the recovery we have that

$$
E\left[X_{t+\Delta}-X_{t} \mid X_{t}\right]=\left(\mu(t)-v \lambda+\mu_{0} h(t)\right) \Delta+o(\Delta) .
$$

Thus, from equation (1) it follows that

$$
\mu(t)=\mu+v \lambda-\mu_{0} h(t) .
$$

Equation (17) shows that an exogenous shock reduces the level of return. The risk (variance) associated with equation (14) is

$$
\begin{aligned}
\operatorname{Var}\left(X_{t+\Delta} \mid X_{t}\right) & =\operatorname{Var}\left[\sigma(t)\left(W_{t+\Delta}-W_{t}\right)\right]+v^{2} \operatorname{Var}\left[j_{1}(t+\Delta)-j_{1}(t)\right] \\
& +\operatorname{Var}\left[j_{2}(t+\Delta) \mid j_{2}(t)=0\right]
\end{aligned}
$$

This gives

$$
\begin{aligned}
\operatorname{Var}\left(X_{t+\Delta} \mid X_{t}\right) & =\left(\sigma^{2}(t)+v^{2} \lambda\right) \Delta+\operatorname{Var}\left[E\left(j_{2}(t+\Delta) \mid j(t)=0\right)\right] \\
& +E\left[\operatorname{Var}\left(j_{2}(t+\Delta) \mid j_{2}(t)=0\right)\right]+o(\Delta) ; \\
\operatorname{Var}\left(X_{t+\Delta} \mid X_{t}\right) & =\left(\sigma^{2}(t)+v^{2} \lambda+\left(\mu_{0}^{2}-\sigma_{0}^{2}\right) h(t)\right) \Delta+o(\Delta) .
\end{aligned}
$$

Similarly, it follows from (2) that

$$
\sigma^{2}(t)+v^{2} \lambda+\left(\mu_{o}^{2}-\sigma_{0}^{2}\right) h(t)=\sigma^{2} ; \quad \sigma^{2}(t)=\sigma^{2}-v^{2} \lambda+\left(\sigma_{0}^{2}-\mu_{0}^{2}\right) h(t) .
$$

If $\sigma_{0}^{2} \geq \mu_{0}^{2}$ the shock thus results in an increase in market volatility alongside a decrease in drift. If $\sigma_{0}^{2} \leq \mu_{0}^{2}$ the exogenous shock actually results in a reduction in volatility. However, irrespective of the effect upon market volatility the shock decreases the rate of return so is still likely to remain bad news for investors. If $\sigma_{0}^{2}=\mu_{0}^{2}$ market volatility remains unaffected.

As in Fry (2012) we choose

$$
h(t)=\omega\left[1-(1+t)^{-\alpha}\right] .
$$

Not only does $h(t)$ in (21) satisfy (13) but the special case $\alpha=0.5$ in (21) recreates empirical power-laws (Sornette et al., 2003; Fry, 2012) and related phenomenology (Crane and Sornette, 2008; Saichev and Sornette, 2010; Sornette and Helmstetter, 2003). Equation (21) also ensures that $h(t)$ is bounded - an important facet of empirical work on related models (Fry, 2010a-b; 2011-12) - and provides a natural test for the presence of an exogenous/endogenous shock (see below). From (21) it follows that

$$
\sigma^{2}(t)=\sigma^{2}-v^{2} \lambda+\beta\left[1-(1+t)^{-\alpha}\right],
$$


where $\beta=\omega\left(\sigma_{0}^{2}-\mu_{0}^{2}\right)$. Equations (21-22) provide a natural way of testing for an exogenous shock in empirical data. The case $\alpha=0$ corresponds to the case where price changes are completely unpredictable and we are left with the benchmark IID model in (5-6).

Exogenous shock. We have that

$$
\frac{\partial \sigma^{2}(t)}{\partial t}=\alpha \beta(1+t)^{-\alpha-1} .
$$

The interpretation depends on the sign of $\alpha \beta$ and hence upon the sign of $\beta$ in (23) since it is assumed that $\alpha>0$ in (22). If $\beta>0$ then then $\sigma^{2}(t)$ increases without bound. This does not appear to be physically or financially reallistic. In contrast, if $\beta<0$ the market recovery becomes the inevitable phase transition between random and deterministic behaviour with

$$
\lim _{t \rightarrow \infty} \sigma^{2}(t)=0 .
$$

This suggests that

$$
\sigma^{2}-v^{2} \lambda+\beta=0 ; \quad \sigma^{2}-v^{2} \lambda=-\beta .
$$

Endogenous shock. Suppose $\alpha<0$. Now $h(t)$ in (21) must remain nonnegative in order to be an admissible hazard function. Thus, in this case we can write

$$
h(t)=\omega\left[(1+t)^{\nu}-1\right],
$$

with $\nu=-\alpha>0$. Hence, we can rewrite (22) as

$$
\sigma^{2}(t)=\sigma^{2}-v^{2} \lambda-\beta\left((1+t)^{\nu}-1\right) .
$$

If $\beta>0 \sigma^{2}(t)$ will become negative for large $t$ - an obvious contradiction. In contrast, if $\beta<0$ we have that

$$
\lim _{t \rightarrow \infty} \sigma^{2}(t)=\infty
$$

Re-writing (28) in terms of the precision, reciprocal of the variance, we see that

$$
\lim _{t \rightarrow \infty} \frac{1}{\sigma^{2}(t)}=0,
$$

and an endogenous shock is again linked to phase-transition phenomena a fundamental imprecision in the quality of the available information with regard to future price movements. 


\subsection{Antibubble-induced speculation in bond yields}

The concept of an antibubble leads to an interesting application to the ongoing euro-crisis. In particular we can show that an antibubble in the price of the underlying asset leads to a bubble in the corresponding Bond yields. Following the standard approach (see e.g. Hillier et al., 2010) write

$$
P(t)=M e^{-y(t) T},
$$

where $y(t)$ is the yield, $T$ is the maturity date, $M$ is the constant value of the bond at maturity and $P(t)$ is the price of the underlying asset. It follows that $X(t)=\ln P(t)$ satisfies

$$
X(t)=\ln M-y(t) T .
$$

Under the equation for an antibubble we have that

$$
d X_{t}=\mu(t) d t+\sigma(t) d W_{t}+v d j(t)
$$

where

$$
\begin{aligned}
\mu(t) & =\mu-v \lambda(t), \\
\sigma^{2}(t) & =\sigma^{2}-v^{2} \lambda(t) .
\end{aligned}
$$

Combining equations (31-33) it follows that the bond yields $y(t)$ satisfy

$$
d y(t)=-\frac{\mu(t)}{T} d t+\frac{\sigma(t)}{T} d W_{t}^{\prime}-\frac{v}{T} d j(t),
$$

where $W_{t}^{\prime}=-W_{t}$. Thus it follows that (34) gives the formula for a speculative bubble since $W_{t}^{\prime} \stackrel{d}{=} W_{t}$.

\subsection{Exogenous shocks to bond yields}

Suppose that an exogenous shock occurs at time 0. Linking the nominal price of the asset to the bond yield, as above, gives

$$
\begin{aligned}
d X_{t} & =-T d y_{t} \\
d y_{t} & =-\frac{1}{T} d X_{t} \\
& =-\frac{1}{T}\left[\mu(t) d t+\sigma(t) d W_{t}+v d j_{1}(t)-d j_{2}(t)\right] .
\end{aligned}
$$




\begin{tabular}{|l|l|l|}
\hline Start Date & $5 / 11 / 2009$ & $8 / 12 / 2009$ \\
\hline$\hat{\alpha}$ & -0.418 & -1.401 \\
e.s.e. $\hat{\alpha}$ & 0.220 & 0.110 \\
$t$-value & 1.901 & 12.783 \\
$p$-value & 0.057 & 0.000 \\
\hline Conclusion & No evidence of & Significant evidence of \\
& an endogenous shock & an exogenous shock \\
\hline
\end{tabular}

Table 3: Results for the test of endogenous vs. exogenous shocks

Prior to the recovery we have that $d J_{2}(t)=0$ and we have that

$$
\begin{aligned}
d y_{t} & =-\frac{1}{T}\left(\left[\mu+v \lambda-\mu_{0} h(t)\right] d t+\beta(1+t)^{-\alpha} d W_{t}+v d j_{1}(t)\right) \\
& =\left[\frac{\mu_{0} h(t)}{T}-(\mu+v \lambda)\right] d t+\frac{\beta}{T}(1+t)^{-\alpha} d W_{t}^{\prime}-\frac{v}{T} d j_{1}(t)
\end{aligned}
$$

where $W_{t}^{\prime}=W_{t}$. Thus equation (36) predicts that the effect of the shock upon the bond yield is an increase in drift coupled with an increase in volatility. Upon estimating (36) econometrically if $\alpha>0$ the shock is classified as exogenous and its long-term effects will eventually dissipate. However, if $\alpha<0$ the shock is classified as endogenous and our model predicts that, unabated, volatility will continue to increase without bound.

\subsection{Empirical application to the Greek/eurozone crisis}

We illustrate our model with an application to the on-going Eurozone crisis. A time series plot of Greek government bond yields is shown below in Figure 2. For a number of years Greek government bond yields appear remarkably stable before spiking upwards in late 2009 following the onset of the European sovereign debt crisis. The precise chronology of the crisis is discussed in Choi et al. (2011). Following established methodology (Sornette et al., 2003; Johansen and Sornette, 2010; Fry, 2012) we test for the presence of an exogenous shock in the first 100 trading days following a putative shock - and cross-check these results with Choi et al. (2011). Results are summarized in Table 3.

Greece's announced plan to cut its budget deficit on 5/11/2009 is heralded by Choi et al. (2011) as the start of the crisis. However, results in Table 3 suggest that this event did not have a statistically significant effect upon Greek bond yields. In contrast, results in Table 3 suggest that 


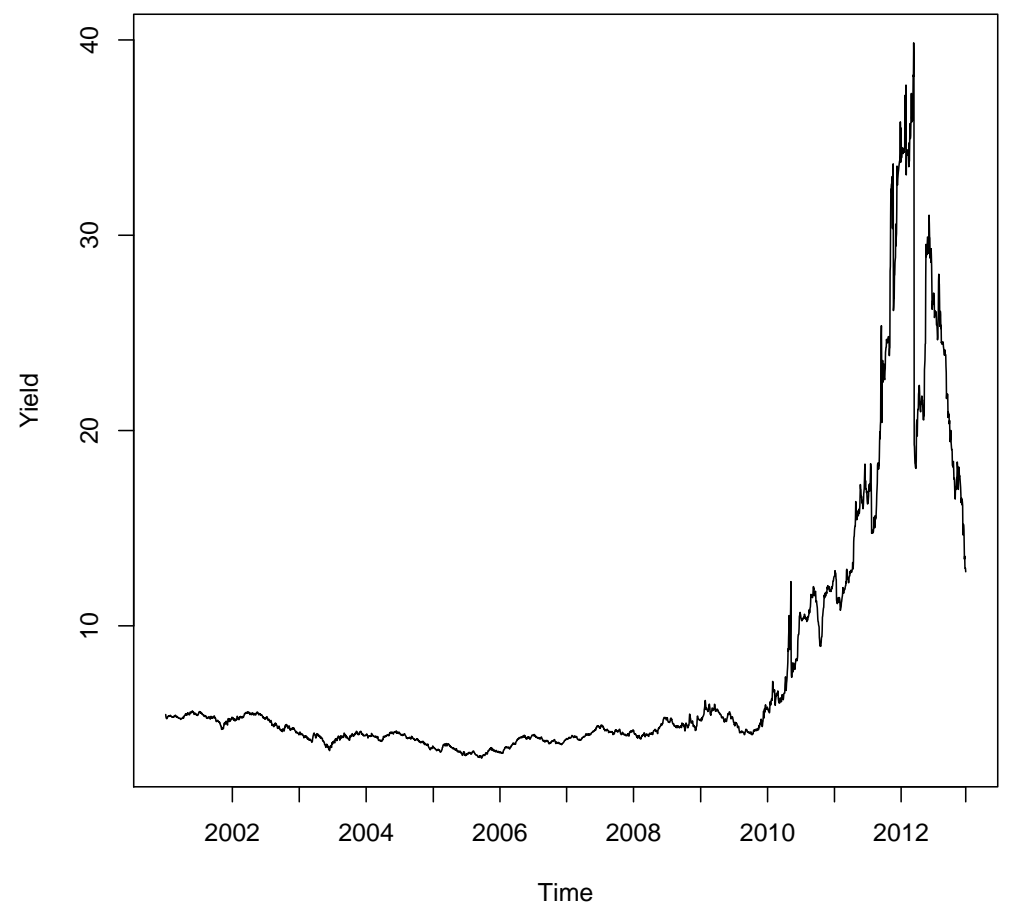

Figure 2: Evolution of Greek government bond yields following adoption of the Euro.

the true start of the crisis occurs on 8/12/2009 when Greece's credit rating was cut by the Fitch credit-rating agency. Moreover, our results classify the Greek crisis as an endogenous shock inextricably linked to fundamental weaknesses in the economies of the Eurozone countries (Blundell-Wagnall, 2011). To verify this interpretation we test for the presence of antibubbleinduced speculation in bond yields according to the model in Section 4.2 from Jan 1st 2010 to December 18th 2012. The maximised log-likelihood for the IID model is -675.2544 , the maximised log-likelihood for the speculative bubble is -623.6579 , giving us a $\chi^{2}$ value of 103.193. Thus, we have significant evidence $(p=0.000)$ that the Euro-crisis is indeed endogenous in nature. 


\section{$5 \quad$ Extensions of market predictions - cycles}

In this section we consider price fluctuations viewed from the perspective of time-varying fluctuations in susceptibility. Thus we continue to borrow important themes and terminology from statistical mechanics (Yeomans, 1992). The motivation for studying this area is as follows. Firstly, cycles appear as a core element of common technical trading strategies (see e.g. Murphy, 1986; Plummer, 2006). However, this is not purely an issue for would-be traders. There is a rich academic literature on business cycles driven by time-varying expectations - see e.g. De Paoli and Zabczyk (2012) and the references therein. Moreover the risk premia that generate the cycles vary over time and are counter-cyclical (Campbell and Cochrane, 1999). Cycles thus emerge as a central issue to both academics and practitioners. Hence, as in Pan et al. (2006), we are interested in links between these two competing theories.

We extend the benchmark IID model (5-6) by writing

$$
d X_{t}=\mu(t) d t+\sigma(t) d W_{t}-v(t) d j_{t},
$$

where $v(t)$ is a periodic function and where $j(t)$ is a Poisson process with parameter $\lambda$. Thus cycles occur as the result of periodic fluctuations in the market's sensitivity to randomly arriving information - i.e. time-varying susceptibility. As above these risk premia vary over time and counter-cyclical (Campbell and Cochrane, 1999). Expanding $v(t)$ as a Fourier series we obtain

$$
v(t)=\sum_{n=1}^{\infty} v_{n} \sin \left(\frac{n \pi t}{L}\right) .
$$

If $v(t)=$ constant equation (37) reduces to the fundamental price/equilibrium model discussed in equation (6). Using the first few terms in the Fourier series in equation (38) we can obtain a low-order approximation for $v(t)$. In applications the appropriate order may be determined using standard techniques from applied statistics. The simplest possible first-order condition gives a sinusoid:

$$
v(t)=v_{1} \sin (\omega t+\phi),
$$

where $\phi$ is a phase constant (Chang and Feigenbaum, 2006). In this case Assumption 1 gives

$$
\mu(t)-\lambda v_{1} \sin (\omega t+\phi)=\mu, \mu(t)=\mu+\lambda v_{1} \sin (\omega t+\phi) .
$$


Similarly, Assumption 2 gives

$$
\sigma^{2}(t)+v_{1}^{2} \lambda \sin ^{2}(\omega t+\phi)=\sigma^{2} ; \sigma^{2}(t)=\sigma^{2}-v_{1}^{2} \lambda \sin ^{2}(\omega t+\phi) .
$$

Finally, the constraint $\min _{t} \sigma^{2}(t)=0$ gives

$$
\lambda v_{1}^{2}=\sigma^{2} ; v_{1}=\sigma / \sqrt{\lambda} .
$$

The end result is a model that appears closely linked to that in (Sørensen, 2003) modelling seasonality in agricultural commodities.

\subsection{Empirical application}

As a practical illustration of our model we apply our model to UK Treasury Gilts. Seasonality in UK Treasury Gilt returns at both monthly and sixmonthly intervals is suggested by Plummer (2006) and appears reasonable (see Figure 3). Using data on returns calculated over first monthly and then six-monthly intervals Plummer (2006) suggests cycles occur at triadic periodicities of $1.78,5.34$ and 16 years. It is therefore of interest to see how our estimates compare to this largely qualitative analysis.

Results for our model are shown below in Table 4. Chi-squared tests show a statistically significant improvement over the benchmark IID model over both time horizons $(p=0.000)$. However, the estimated period of the cycle, $\hat{L}$, holds some additional importance. Firstly, using monthly data the estimate of $L$ obtained is extremely close to 1 . This suggests that rather than genuine periodicity what we have is auto-correlation between UK Treasury Gilt returns in successive periods. Using the 6-monthly data we estimate the length of the cycle to be around 2.5 years. This appears to be roughly in line with a Kitchin inventory cycle of around 3 years but is significantly different from the original interpretation of Plummer (2006). Thus we conclude that the measurement of cycles in technical seems imprecise and should be viewed cautiously. These results also tally with a common theme that emerges in the academic literature on time series econometrics - namely that it is often better to use lower frequency data to identify cyclical variations and regime switching (see e.g. Walid et al., 2011).

\section{$6 \quad$ Extensions of market predictions - support and resistance lines}

Support and resistance lines, and technical analysis more generally, is the subject of an extremely large literature by academics and practitioners alike. 

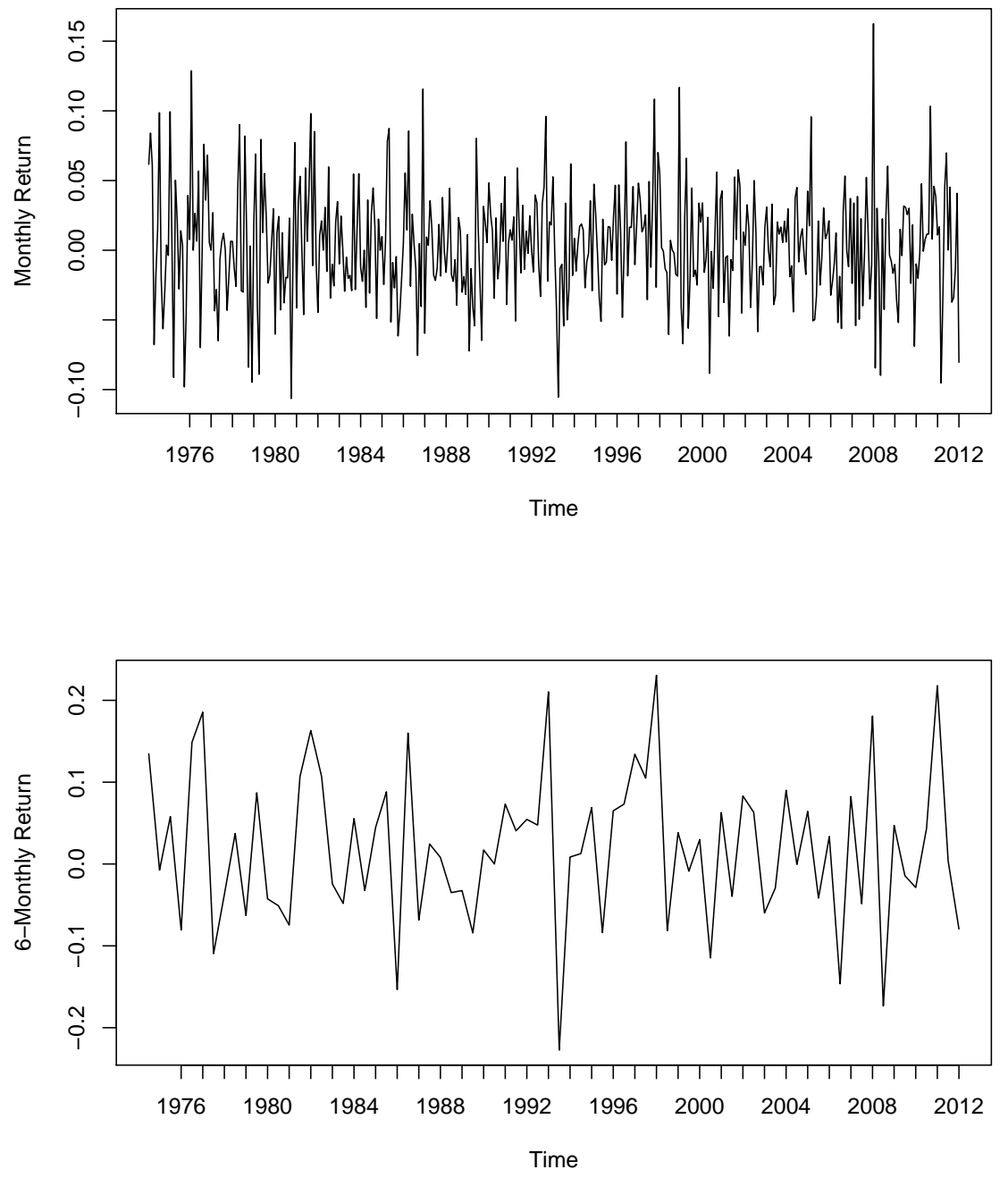

Figure 3: Returns for Treasury Stock 2.5\% irredeemable. Left panel: Monthly returns. Right panel: Six-monthly returns

Further, the use of technical analysis is simply too widespread, and therefore too important, to ignore. For instance evidence suggests that technical analysis has been used by over $90 \%$ of participants in the Foreign Exchange 


\begin{tabular}{|l|l|l|}
\hline & Monthly Returns & Six-Monthly Returns \\
\hline Benchmark (iid) model & 73.360 & 817.556 \\
Cyclical model & 81.371 & 1556.223 \\
\hline \hline$\hat{L}$ & 1.0100 months & 2.45 years \\
$95 \%$ C.I. & $(1.0097,1.0103)$ months & $(2.43,2.47)$ years \\
\hline
\end{tabular}

Table 4: Results for the cyclical model: monthly and six-monthly returns

market (Osler, 2000; Allen and Taylor, 1992; Lui and Mole, 1998). A wide ranging review of academic research on technical analysis - including different tools and techniques across a diverse range of markets - can be found in Park and Irwin (2007). Osler (2000) is also interesting for its discussion of the practical role played by support and resistance lines in real-world Foreign Exchange markets. There is clearly much work that can be conducted on related themes. Here, we restrict to a discussion of support and resistance lines.

An example of support and resistance lines is shown in Figure 4. Support levels occur when prices are about to cross a de facto localised lower threshold. Once the threshold is reached the decline is, at least temporarily, halted and the price turns back again. Resistance levels represent the opposite process and occur when prices are about to pass a de facto upper bound. Similarly, once this threshold is reached prices fall back down below the resistance line. Support and resistance lines can be modelled as follows.

\subsection{Support lines}

Support lines emerge as a key theme of the practitioner literature on technical analysis as an effective lower bound on the price of an asset (see e.g. Plummer, 2006). Suppose we have a support line around the point $a$. Our equation for the price of an asset becomes

$$
d X_{t}=\mu(t) d t+\sigma(t) d W_{t}-\left[v_{1}+v_{2} 1_{\left\{\left(X_{t}<a\right)\right\}}\right] d j_{1}(t) .
$$

Equation (42) shows that once the support line is exceeded the market becomes increasingly nervous. The negative effect of any incoming information is magnified. In order to maintain an equilibrium the drift function $\mu(t)$ and the volatility $\sigma^{2}(t)$ have to adapt in order to compensate a representative investor for bearing the additional risk involved. Assumption 1 gives

$$
\mu(t)=\mu+\lambda v_{1}+\lambda v_{2} 1_{\left\{\left(X_{t}<a\right)\right\}} .
$$


Similarly, Assumption 2 gives

$$
\sigma^{2}(t)=\sigma^{2}-\lambda\left[v_{1}+v_{2} 1_{\left\{\left(X_{t}<a\right)\right\}}\right]^{2} .
$$

\subsection{Resistance lines}

Support lines emerge as a key theme of the practitioner literature on technical analysis as an effective upper bound on the price of an asset (Plummer, 2006). Suppose we have a support line around the point $b$. Our equation for the price of an asset becomes

$$
d X_{t}=\mu(t) d t+\sigma(t) d W_{t}-\left[v_{1}-v_{2} 1_{\left\{X_{t}>b\right\}}\right] d j_{1}(t) .
$$

Equation (42) shows that once the support line is exceeded the market anticipates future price rises. The positive (i.e. inflationary) effect of any incoming information is magnified. In order to maintain an equilibrium the drift function $\mu(t)$ and the volatility $\sigma^{2}(t)$ have to adapt as above. Assumption 1 gives

$$
\mu(t)=\mu+\lambda v_{1}-\lambda v_{2} 1_{\left\{X_{t}>b\right\}} .
$$

Similarly, Assumption 2 gives

$$
\sigma^{2}(t)=\sigma^{2}-\lambda\left[v_{1}-v_{2} 1_{\left\{X_{t}>b\right\}}\right]^{2} .
$$

\subsection{Practical application}

One of the examples of support and resistance lines cited in Griffis and Epstein (2009) is that of Baxter International Incorporated (BAX) quoted on the New York stock exchange. Using qualitative methods Griffis and Epstein (2009) fit a support line at 34.5 dollars and a resistance line at 38.0 dollars respectively. A plot of the share price from January to August 2006 is shown in Figure 4 and appears suggestive. However, rigorous statistical results shown in Table 5 refute this interpretation. Using a $\chi^{2}$ test evidence is found for a support line $(p=0.000)$ but no evidence is found for an additional resistance line $(p=0.136)$. A resistance line it estimated to occur at 37.74 dollars - $95 \%$ confidence interval $(37.739,37.741)$ dollars.

In conclusion, a wide range of evidence exists to suggest that technical trading strategies can indeed yield profits (Park and Irwin, 2007). Further, technical analysis doubtless contains some useful concepts (Osler, 2000). Here, we find evidence for a resistance line - although our estimate is significantly lower than practitioner estimates. In contrast to the practitioner 
interpretation we find no evidence of a support line - suggesting that practitioners may under-estimate the extent of the true risks involved with stock trading. Since we find no evidence of a support line there appears to be no obvious reason why the price may not drop below 34.5 dollars.

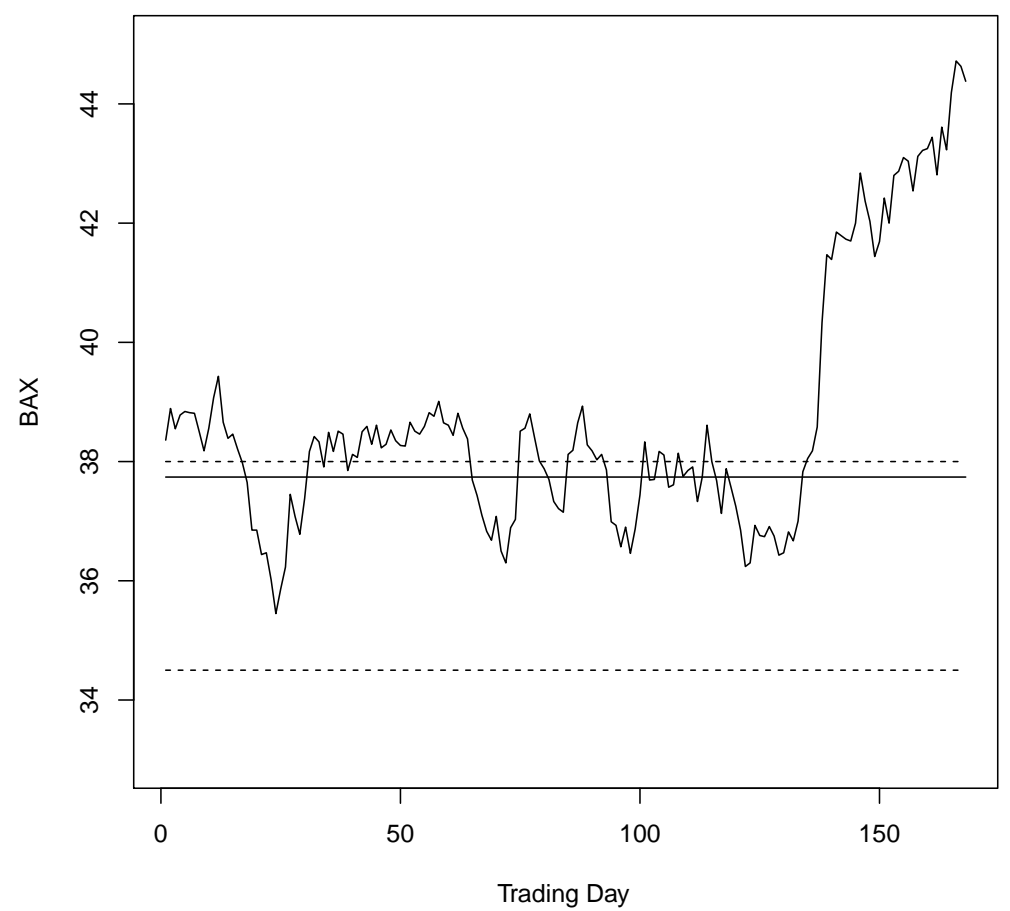

Figure 4: Putative support and resistance lines identified by practitioners using a qualitative approach at 34.5 and 38.0 dollars respectively compared to daily closing prices. The solid horizontal line shows the resistance line identified here using a mathematical approach.

\section{Conclusions}

In this paper we have continued to extend the analogy between financial crashes and phase transition phenomena in statistical mechanics (see e.g. Johansen et al., 2000). However, whilst this literature contains important 


\begin{tabular}{|l|l|}
\hline Model & Maximised Log-likelihood \\
\hline Equilibrium (background risk) model & 505.0302 \\
Resistance line model & 623.1823 \\
Support and resistance line model & 625.1806 \\
\hline
\end{tabular}

Table 5: Results of the test for support and resistance lines in the Microsoft share price.

insights and techniques and numerous worthy contributions there have been simple and obvious failings in the popularly applied methods. In short, markets operate by balancing risk and return (Markowitz, 1971). This idea is not new but appears to have been fundamentally over-looked by academics and practitioners alike. Crucially, rates of return are only part of the story. Risk is also critically important. Once this crucial missing piece of the puzzle is added it can be explicitly shown that, from a physical perspective, changes in market regime represent a phase transition between random and deterministic behaviour in prices. This idea is known to apply to endogenous and exogenous crashes (Fry, 2012) but can also be extended to include elementary technical trading strategies - here support and resistance lines and business cycles. We are thus lead to the intriguing possibility that tools and techniques from statistical physics may assist practitioners.

A better physical model also leads directly to a better econometric model. Not only is the scope of our model significantly expanded, as discussed above, but in formulating our model we are left with fewer unknown parameters to estimate than the competing class of log-periodic models. Estimation is possible using a maximum likelihood approach and we can also naturally incorporate empirically observable features of real asset prices into our model - namely skewness and heavy tails. Incorporating these features into our model should lead to more refined tests for bubbles and other short-term deviations from long-run equilibria (Fry, 2008).

Our approach has attracted attention from market regulators and underscores the practical utility of the physical sciences - even to non-traditional areas. The potential relevance to on-going events and policy debates is clearly apparent. Firstly, we analyse real-estate bubbles an obvious underlying feature of the on-going crisis. In particular, we are able to show that UK house prices are intrinsically volatile and have been subject to a various bubble and antibubble episodes over the last three decades. Most recently, we find evidence of a bubble from 1999-2006 and of an antibubble from 2008-11. Secondly, we apply our endogenous shock and antibubble models 
to the on-going Greek crisis in the Eurozone. To elucidate the chronology in Choi et al. (2011) we can show that the current crisis represents an endogenous shock triggered around the time of the Fitch ratings' downgrade on December 8th 2009.

Our approach may have further relevance to market participants even outside periods of crisis. Using our model we scrutinise popular technical trading strategies - namely cycles (see e.g. Plummer, 2006) and support and resistance lines (Griffis and Epstein, 2009). Whilst some technical strategies may be profitable (Park and Irwin, 2007) and may provide genuinely useful price information (Osler, 2000) the predictions of these predominantly qualitative approaches appear somewhat vague. Our estimated cycle lengths do not match estimates in Plummer (2006). Further, our analysis of support and resistance lines identified in Griffis and Epstein (2009) suggests that some common technical analysis strategies may give traders a misplaced sense of confidence and overlook some of the inherent risks involved.

In conclusion, our paper contributes to a rich interplay between physics and finance and to wider narratives in contemporary accounting and business research. Having identified a mathematical approach hitherto largely overlooked by the literature future work will consider additional risk management and policy applications. Future work will address further modelling of business cycles and will explore additional links between academic and practitioner finance - perhaps one day contributing towards a unified theory (Pan et al., 2006).

\section{References}

[1] Allen, H. and Taylor, M. P. (1992) The use of technical analysis in the Foreign Exchange market. Journal of International Money and Finance 11 304-14.

[2] Blundell-Wagnall, A. (2011) Solving the financial and sovereign debt crisis in Europe. OECD Journal: Financial Market Trends 2011 (2) $1-23$.

[3] Bouchaud, J-P., and Potters, M. (2003) Theory of financial risk and derivative pricing. From statistical physics to risk management, second edition. Cambridge University Press, Cambridge.

[4] Bree, D., Challet, D. and Perrano, P. P. (2013) Prediction accuracy and sloppiness of log-periodic functions. Quantitative Finance 13 275-280. 
[5] Bree, D. and Joseph, N. L. (2013). Fitting the log periodic power law to financial crashes: a critical analysis. Preprint.

[6] Campbell, J. Y., and Cochrane, J. H. (2000) Explaining the poor performance of consumption-based asset pricing models. Journal of Finance $552863-2878$.

[7] Chang, G., and Feigenbaum, J. (2006) A Bayesian analysis of logperiodic precursors to financial crashes. Quantitative Finance 6 15-36.

[8] Chang, G., and Feigenbaum, J. (2008) Detecting log-periodicity in a regime-switching model of stock returns. Quantitative Finance 8 723738.

[9] Choi, S. J., Gulati, M., and Posner, E. A. (2011) Pricing terms in sovereign debt contracts: a Greek case study with implications for the European crisis resolution mechanism. Capital Markets Law Journal 6 163-187.

[10] Cont, R. and Tankov, P. (2004). Financial modelling with jump processes. Chapman and Hall/CRC, Boca Raton London Hiedelberg Washington DC.

[11] Crane, R. and Sornette, D. (2008) Robust dynamic classes revealed by measuring the response function of a social system. PNAS 105 1564915653.

[12] De Paoli, B. and Zabczyk, P. (2012) Why do risk premia vary over time? A theoretical investigation under habit formation. Macroeconomic Dynamics 16 252-266.

[13] Feigenbaum, J. (2001a) A statistical analysis of log-periodic precursors to financial crashes. Quantitative Finance 1 346-360.

[14] Feigenbaum, J. (2001b) More on a statistical analysis of log-periodic precursors to financial crashes. Quantitative Finance 1 527-532.

[15] Fry, J. M. (2008) Statistical modelling of financial crashes. Unpublished PhD thesis, Department of Probability and Statistics, University of Sheffield.

[16] Fry, J. M. (2010a) Bubbles and crashes in finance: A phase transition from random to deterministic behaviour in prices. Journal of Applied Research in Finance 2 131-137. 
[17] Fry, J. M. (2010b) Modelling bubbles and crashes in economics: With an application to English house prices. VDM Publishing, Saarbrucken.

[18] Fry, J. M. (2011) Gaussian and non-Gaussian models for financial bubbles via econophysics. Hyperion International Journal of Econophysics and New Economy 4 7-22.

[19] Fry, J. M. (2012) Endogenous and endogenous market crashes as phase transitions in complex financial systems. European Physical Journal B 85405.

[20] Geraskin, O. and Fantazzini, D. (2011) Everything you always wanted to know about log-periodic power laws for bubble modelling but were afraid to ask. European Journal of Finance (forthcoming)

[21] Griffis, M., and Epstein, L. (2009) Trading for dummies, Second Edition. Wiley, Hoboken, New Jersey.

[22] Hillier, D., Ross, S., Westerfeld, R., Jaffe, J., and Jordan, B. (2010) Corporate Finance, fourth edition. McGraw-Hill, Maidenhead, Berkshire.

[23] Jiang, Z-Q., Zhou, W-X., Sornette, D., Woodard, R., Bastiaenesen, K. and Cauwels, P. (2010) Bubble diagnosis and prediction of the 20052007 and 2008-2009 Chinese stock market bubbles. Journal of Economic Behaviour and Organization 74 149-162.

[24] Johansen, A., Ledoit, O., and Sornette, D. (2000) Crashes as critical points. International Journal of Theoretical and Applied Finance 3 219255.

[25] Johansen, A. and Sornette, D. (2010) Shocks, crashes and bubbles in financial markets. Brussels Economic Review 53 201-253.

[26] Kurz-Kim, J. R. (2012) early warning indicator for financial crashes using the log-periodic power law. Applied Economics Letters 19 14651469 .

[27] Lillo, F., and Mantegna, R. (2003) Power-law relaxation in a complex system: Omori law after a financial market crash. Physical Review E 68016119.

[28] Lin, L. and Sornette, D. (2011) Diagnostics of rational expectations financial bubbles with stochastic mean-reverting termination imes. European Journal of Finance (forthcoming) 
[29] Lo, A., Mamaysky, H. and Wang, J. (2000) Foundations of technical analysis: computational algorithms, statistical inference and empirical implementation. Journal of Finance 55 1705-1765.

[30] Lui, Y-H., and Mole, D. (1998) The use of fundamental and technical analyses by Foreign Exchange dealers: Hong Kong evidence. Journal of International Money and Finance 17 535-545.

[31] Markowitz, H.M. (1971) Portfolio Selection: Efficient Diversification of Investments, Second Edition. Blackwell, Malden, Massachussets.

[32] Merton, R. (1976) Options pricing when underlying stock returns are discontinuous. Journal of Financial Economics 3 125-144.

[33] Murphy, J. J. (1986) Technical analysis of the futures markets: A comprehensive guide to trading methods and applications. Prentice Hall, New York.

[34] Osler, C. (2000) Support for resistance: technical analysis and intraday exchange rates. FRBNY Economic Policy Review July 53-68.

[35] Pan, H., Sornette, D. and Kortanek, K. (2006) Intelligent finance - an emerging direction. Quantitative Finance 6 273-277.

[36] Park, C-H., and Irwin, S. H. (2007) What do we know about the profitability of technical analysis? Journal of Economic Surveys 21 786-826

[37] Peston, R. and Knight, L. (2012) How do we fix this mess? The economic price of having it all and the route to lasting prosperity. Hodder and Stoughton, London.

[38] Plummer, T. (2006) Forecasting financial markets. The psychology of successful investing. Kogan Page, London.

[39] Pryce, V. (2012) Greekonomics: The Euro crisis and why politicians don't get it. Biteback, London.

[40] Saichev, A., and Sornette, D. (2010) Generation-by-generation dissection of the response function in long memory epidemic processes. European Physical Journal B 75 343-355.

[41] Sørensen, C. (2003) Modeling seasonality in agricultural commodity futures. The Journal of Futures Markets 22 393-426. 
[42] Sornette, D. and Helmstetter, A. (2003) Endogeneous Versus Exogeneous Shocks in Systems with Memory. Physica A 318 577-591.

[43] Sornette, D., Malevergne, Y. and Muzy, J. F. (2003) What causes crashes? Risk 2 67-71.

[44] Sornette, D., and Zhou, W-X. (2006) Predictability of large future changes in major financial indices. International Journal of Forecasting 22 153-168.

[45] Sornette, D. (2003) Why Stock Markets Crash: Critical events in Complex Financial Systems. Princeton University Press, Princeton.

[46] Walid, C., Chaker, A., Masood, O. and Fry, J. (2011) Stock market volatility and exchange rates in emerging countries: A Markov-state switching approach. Emerging Markets Review 12 272-292.

[47] WHite, M., and Fry, J. M. (2013) Bubbles and antibubbles in UK house prices. Preprint.

[48] Yan, W., Woodard, R., and Sornette, D. (2012) Diagnosis and prediction of rebounds in financial markets. Physica A 391 1361-1380.

[49] Yeomans, J. M. (1992) Statistical mechanics of phase transitions. Oxford University Press, Oxford.

[50] Zeira, J. (1999) Informational overshooting, booms and crashes. Journal of Monetary Economics 43 237-257.

[51] Zhou, W-X., and Sornette, D. (2004) Antibubble and prediction of China's stock market and real-estate. Physica A 337 243-268.

[52] Zhou, W-X., and Sornette, D. (2005) Testing the Stability of the 20002003 US Stock Market "Antibubble". Physica A 348 428-452.

[53] Zhou, W-X., and Sornette, D. (2008) Analysis of the real estate market in Las Vegas: Bubble, seasonal patterns, and prediction of the CSW indexes. Physica A 387 243-260.

[54] Zhou, W-X., and Sornette, D. (2009) A case study of speculative financial bubbles in the South African stock market 2003-2006. Physica A 388 869-880. 